\title{
NON-CYCLIC ALGEBRAS OF DEGREE AND EXPONENT FOUR*
}

\author{
BY
}

\section{A. ADRIAN ALBERT}

1. Introduction. I have recently $\dagger$ proved the existence of non-cyclic normal division algebras. The algebras I constructed are algebras $A$ of order sixteen (degree four, so that every quantity of $A$ is contained in some quartic sub-field of $A$ ) containing no cyclic quartic sub-field and hence not of the cyclic (Dickson) type. But each $A$ is expressible as a direct product of two (cyclic) algebras of degree two (order four). Hence the question of the existence of non-cyclic algebras not direct products of cyclic algebras, and therefore of essentially more complex structures than cyclic algebras, has remained unanswered.

The exponent of a normal division algebra $A$ is the least integer $e$ such that $A \bullet$ is a total matric algebra. A normal division algebra of degree four has exponent two or four according as it is or is not expressible as a direct product of algebras cf degree two. $\ddagger$ I shall prove here that there exist non-cyclic normal division algebras of degree and exponent four, algebras of a more complex structure than any previously constructed normal division algebras.

2. Algebras of order sixteen. We shall consider normal simple algebras of order sixteen (degree four) over a field $K$. Algebra $A$ has a quartic sub-field $K(u, v)$ where

$$
u^{2}=\rho, \quad v^{2}=\sigma \quad(\rho, \sigma \text { in } K),
$$

such that neither $\rho, \sigma$, nor $\sigma \rho$ is the square of any quantity of $K$. Algebra $A$ contains quantities

$$
j_{1}, j_{2}, j_{3}=j_{1} j_{2}
$$

such that

$$
\begin{aligned}
& j_{1} u=u j_{1}, \quad j_{1} v=-v j_{1}, \quad j_{1}{ }^{2}=g_{1}=\gamma_{1}+\gamma_{2} u \neq 0\left(\gamma_{1}, \gamma_{2} \text { in } K\right), \\
& j_{2} v=v j_{2}, \quad j_{2} u=-u j_{2}, \quad j_{2}{ }^{2}=g_{2}=\gamma_{3}+\gamma_{4} v \neq 0\left(\gamma_{3}, \gamma_{4} \text { in } K\right), \\
& j_{2} j_{1}=\alpha j_{3}, \quad j_{3}{ }^{2}=g_{3}=\gamma_{5}+\gamma_{6} u v\left(\gamma_{5}, \gamma_{6} \text { in } K\right),
\end{aligned}
$$

* Presented to the Society, August 31, 1932; received by the editors June 9, 1932.

$\dagger$ In a paper published in the Bulletin of the American Mathematical Society, June, 1932. (Designated by Albert 1.)

† See Theorem 6 of my Normal division algebras of degree four, etc., these Transactions, vol. 34 (1932), pp. 363-372. (Designated by Albert 2.) 


$$
\alpha=\frac{\gamma_{5}-\gamma_{6} u v}{\left(\gamma_{1}+\gamma_{2} u\right)\left(\gamma_{3}-\gamma_{4} v\right)} .
$$

A necessary and sufficient condition that $A$ be associative is that

$$
\gamma_{5}^{2}-\gamma_{6}^{2} \sigma \rho=\left(\gamma_{1}^{2}-\gamma_{2}^{2} \rho\right)\left(\gamma_{3}^{2}-\gamma_{4}^{2} \sigma\right) \text {. }
$$

A necessary and sufficient condition* that $A$ be not expressible as a direct product of two algebras of degree two (that is, have exponent four) is that the equation

$$
\alpha_{1}^{2}-\alpha_{2}^{2} \sigma-\left(\gamma_{1}^{2}-\gamma_{2}^{2} \rho\right) \alpha_{3}^{2}=0
$$

be impossible for any $\alpha_{1}, \alpha_{2}, \alpha_{3}$ not all zero and in $K$.

Algebra $\uparrow A$ has a sub-algebra $B=\left(1, v, j_{1}, v j_{1}\right)$ over $K(u)$. This algebra is a generalized quaternion algebra and it is well known that $B$ is a division algebra if and only if

$$
g_{1} \neq a_{1}^{2}-a_{2}^{2} \sigma
$$

for any $a_{1}$ and $a_{2}$ in $K(u)$. But if $a_{1}=\alpha_{1}+\alpha_{2} u, a_{2}=\alpha_{3}+\alpha_{4} u$, the equation $g_{1}=a_{1}^{2}-a_{2}^{2} \sigma$ implies that $\gamma_{1}+\gamma_{2} u=\left[\alpha_{1}^{2}+\alpha_{2}^{2} \rho-\sigma\left(\alpha_{3}^{2}+\alpha_{4}^{2} \rho\right)\right]+2\left(\alpha_{1} \alpha_{2}-\sigma \alpha_{3} \alpha_{4}\right) u$ so that $\gamma_{1}=\alpha_{1}^{2}+\alpha_{2}^{2} \rho-\sigma\left(\alpha_{3}^{2}+\alpha_{4}^{2} \rho\right)$. We have now

THEOREM 1. A sufficient condition that $B$ be a division algebra is that the quadratic form

$$
Q=\left(\alpha_{1}^{2}+\alpha_{2}^{2} \rho\right)-\sigma\left(\alpha_{3}^{2}+\alpha_{4}^{2} \rho\right)-\gamma_{1} \alpha_{5}^{2}
$$

in the variables $\alpha_{1}, \cdots, \alpha_{5}$ shall not vanish for any $\alpha_{1}, \cdots, \alpha_{5}$ not all zero and in $K$.

For if the sufficient condition of Theorem 1 were satisfied and yet $B$ were not a division algebra we would have $\gamma_{1}=\alpha_{1}^{2}+\alpha_{2}^{2} \rho-\sigma\left(\alpha_{3}^{2}+\alpha_{4}^{2} \rho\right)$ so that $Q=0$ for $\alpha_{1}, \alpha_{2}, \alpha_{3}, \alpha_{4}$ in $K$ and $\alpha_{5}=1$, a contradiction.

It is also known $\ddagger$ that, when $B$ is a division algebra, $A$ is also a division algebra if and only if there is no quantity $X$ in $B$ for which

$$
g_{2}=X^{\prime} X
$$

where if $X=b+d j_{1}$ then $X^{\prime}=b(-u)+d(-u) \alpha j_{1}$ with $a$ and $b$ of course in $K(u, v)$.

* See Albert 2.

† For the properties of this section see my paper in these Transactions, vol. 32 (1930), pp. 171195. (Designated hereafter by Albert 3.)

† See L. E. Dickson's Algebren und ihre Zahlentheorie, p. 64, for both the condition that $B$ be a division algebra and $A$ be a division algebra. 
I have proved* that

$$
\left(b j_{2}\right)^{2}=f_{3}+f_{4} v, \quad\left(d j_{3}\right)^{2}=f_{5}+f_{6} u v,
$$

where if

$$
b=\beta_{1}+\beta_{2} v+\left(\beta_{3}+\beta_{4} v\right) u, \quad d=\delta_{1}+\delta_{2} u v+\left(\delta_{3}+\delta_{4} u v\right) u
$$

and

$$
\begin{array}{ll}
b_{1}=\beta_{1}{ }^{2}+{\beta_{2}}^{2} \sigma-\rho\left({\beta_{3}}^{2}+{\beta_{4}}^{2} \sigma\right), & b_{2}=2\left(\beta_{1} \beta_{2}-\rho \beta_{3} \beta_{4}\right), \\
d_{1}=\delta_{1}{ }^{2}+\delta_{2}{ }^{2} \sigma \rho-\rho\left(\delta_{3}{ }^{2}+\delta_{4}{ }^{2} \sigma \rho\right), & d_{2}=2\left(\delta_{1} \delta_{2}-\sigma \rho \delta_{3} \delta_{4}\right),
\end{array}
$$

then

$$
\begin{aligned}
& f_{3}=b_{1} \gamma_{3}+b_{2} \sigma \gamma_{4}, \quad f_{4}=b_{1} \gamma_{4}+b_{2} \gamma_{3}, \\
& f_{5}=d_{1} \gamma_{5}+d_{2} \sigma \rho \gamma_{6},
\end{aligned}
$$

I have also shown that if $g_{2}=X^{\prime} X$ then

$$
f_{4}=f_{6}=0, f_{3}+f_{5}=\gamma_{3}^{2}-\gamma_{4}^{2} \sigma .
$$

But then $\gamma_{3} b_{2}=-\gamma_{4} b_{1}, \gamma_{5} d_{2}=-\gamma_{6} d_{1}$, so that from $\left(16_{2}\right),(15)$,

$$
\gamma_{3} \gamma_{5}\left(\gamma_{3}^{2}-\gamma_{4}^{2} \sigma\right)=\left(\gamma_{3}^{2}-\gamma_{4}^{2} \sigma\right) \gamma_{5} b_{1}+\left(\gamma_{5}^{2}-\gamma_{6}{ }^{2} \sigma \rho\right) \gamma_{3} d_{1} .
$$

If $A$ is associative then (6) is satisfied. Also $g_{2} \neq 0$ so that $g_{2}(-v) \neq 0, \gamma_{3}^{2}-\gamma_{4}^{2} \sigma$ $\neq 0$. Then $(17)$ is equivalent to

$$
\gamma_{3} \gamma_{5}=\gamma_{5} b_{1}+\gamma_{3} d_{1}\left(\gamma_{1}^{2}-\gamma_{2}^{2} \rho\right) .
$$

As in the proof of Theorem 1 we have immediately

Theorem 2. $A$ sufficient condition that $A$ with division sub-algebra $B$ be $a$ division algebra is that the quadratic form

$$
\begin{aligned}
Q \equiv \gamma_{5}\left[\left(\alpha_{1}^{2}+\right.\right. & \left.\left.\alpha_{2}^{2} \sigma\right)-\rho\left(\alpha_{3}^{2}+\alpha_{4}^{2} \sigma\right)\right] \\
& +\gamma_{3}\left(\gamma_{1}^{2}-\gamma_{2}^{2} \rho\right)\left[\left(\alpha_{5}^{2}+\alpha_{6}^{2} \sigma \rho\right)-\rho\left(\alpha_{7}{ }^{2}+\alpha_{8}{ }^{2} \sigma \rho\right)\right]-\gamma_{3} \gamma_{5} \alpha_{9}{ }^{2}
\end{aligned}
$$

shall not vanish for any $\alpha_{1}, \cdots, \alpha_{9}$ not all zero and in $K$.

3. Algebras over $K(q)$. Let $L=K(q)$ be a quadratic field over $K$ where

$$
q^{2}=\delta=\delta_{1}{ }^{2}+\delta_{2}{ }^{2} \quad\left(\delta_{1} \text { and } \delta_{2} \text { in } K\right) .
$$

It is well known that if $K$ contains no quantity $k$ such that $k^{2}=-1$ then every cyclic quartic field over $K$ contains a quadratic sub-field $L$ of the above type. Hence a sufficient condition that an algebra of degree four be non-cyclic is that $A$ contain no quadratic sub-field $L$ as above. But also $A$ contains no sub-

* Albert 3, p. 178. 
field equivalent to any given quadratic field $L$ if and only if $A \times L$ is a division algebra.* Hence we have

THEOREM 3. If no $k$ in $K$ has the property $k^{2}=-1$, a sufficient condition that a normal simple algebra $A$ of order sixteen over $K$ be a non-cyclic normal division algebra is that $A \times L$ be a division algebra for every quadratic field $L=K(q)$,

$$
q^{2}=\delta=\delta_{1}{ }^{2}+\delta_{2}^{2} \quad\left(\delta_{1} \text { and } \varepsilon_{2} \text { in } K\right) .
$$

We shall apply Theorem 3 as follows. We shall choose a particular field of reference, $K$. We shall then define $A$ by a choice of $\rho, \sigma, \gamma_{1}, \cdots, \gamma_{6}$. Then also $A \times L$ is evidently a normal simple algebra (of the same kind as $A$ over $K)$ over $L$ when we show that neither $\rho, \sigma$, nor $\sigma \rho$ is the square of any quantity of $L$ (not merely $K$ ). We shall then prove that $A$ (not $A \times L$ which can have exponent two) has exponent four, while $A \times L$ is a division algebra. This latter step will be an application of Theorems 1 and 2 applied to $A \times L$ over $L$. The algebras $A$ over $K$ will be non-cyclic algebras of exponent four by Theorem 3 .

4. The field $K$. Let $F$ be any real number field, and let $x, y$, and $z$ be independent marks (indeterminates). The field $F(x, y, z) \equiv K$ is a function field consisting of all rational functions with (real) coefficients in $F$ of $x, y, z$. We shall deal with quadratic forms $Q$ and equations $Q=0$ so that we shall always be able to delete denominators and hence take our quantities in

$$
J=F[x, y, z]
$$

the domain of integrity consisting of all polynomials in $x, y, z$ with coefficients in $F$. We shall of course also consider the domains $F[x], F[x, y]$, etc.

Consider a field $K(q)$ as in $\$ 3$. It is evident that the quantity $q$ defining such a quadratic field may always be chosen so that $\delta, \delta_{1}, \delta_{2}$ are in $J$. Also in a quadratic form $Q=0$ with coefficients in $J$ and variables over $K(q)$ we may always take the variables to be in the domain of integrity $J[q]$ of all quantities of the form

where $a$ and $b$ are in $J$.

$$
a+b q
$$

Every quantity $a=a(x, y, z)$ of $J$ has a highest power $z^{n}$ with coefficient in $F[x, y]$ not identically zero. We shall call $n$ the $z$-degree of $a$, the coefficient of $z^{n}$ the $z$-leading coefficient of $a$. Similarly $a$ has an $x$-degree, $y$-degree, $x$ leading coefficient, $y$-leading coefficient. A restriction of the $z$-degree of a certain expression and its z-leading coefficient evidently does not affect its $x$-degree, etc.

* Cf. Albert 1. 
If the coefficient of $z^{n}$ above is $b(y, x)$ and the coefficient of the highest power $y^{m}$ of $y$ in $b$ is $c(x)$, then $m$ is called the $(z, y)$-degree of $a, c(x)$ the $(z, y)$-leading coefficient of $a$. Finally the degree of $c(x)$ is the $(z, y, x)$-degree of $a$, its leading coefficient in $F$, the $(z, y, x)$-leading coefficient of $a$.

We have similarly $(x, y, z)$-degree and leading coefficient, etc. Using these definitions an elementary result is

LEMMA 1. The field $K$ contains no quantity $k$ such that $k^{2}=-1$.

For let $k^{2}=-1$. Then $r k=s$, where $r$ and $s$ are in $J$ and are both not zero. It follows that $s^{2}=-r^{2}$. The $(x, y, z)$-leading coefficient of $s^{2}$ is evidently a real square and is positive, that of $-s^{2}$, negative so that the polynomial identity $r^{2}=-s^{2}$ is impossible.

Lemma 2. There exist quantities $\lambda, \mu$ in $F[x, y]$ such that $\lambda^{2}+\mu^{2}$ is not the square of any quantity of $F(x, y)$.

We prove the above lemma with the example $\lambda=x, \mu=y$. If $x^{2}+y^{2}=b^{2}$, where $b$ is a rational function of $x$ and $y$, it is evident that $b$ must be a polynomial in $x$ and $y$. For the square of a rational function in its lowest terms and with denominator not unity is never a polynomial. Hence we may put $b=b_{1} x+b_{2}$ where $b_{2}$ is in $F[y], b_{1}$ merely in $F[x, y]$. Then $x^{2}+y^{2}=b_{1}^{2} x^{2}$ $+2 b_{1} b_{2} x+b_{2}^{2}$ identically in $x$ and $y$. It follows that $b_{2}^{2}=y^{2}, b_{2}= \pm y$. Then $x^{2}=b_{1}^{2} x^{2} \pm 2 b_{1} x y$. Hence $b_{1}$ divides $x$ and is a power of $x$. But then $\pm\left(2 b_{1}\right) y$ $=x-b_{1}^{2} x$ in $F[x], b_{1}$ in $F(x)$, which is impossible.

5. The $S$-polynomials. The quadratic forms (9), (19) over $L$ shall be treated as follows. If $Q=\sum \alpha_{i}^{2} \lambda_{i}$ with $\lambda_{i}$ in $J$ (not in $J[q]$ ) vanishes for $\alpha_{i}$ in $L$ and not all zero, then obviously, by multiplying $Q$ by the square of the least common denominator, not zero and in $J$, of the $\alpha_{i}=\alpha_{i 1}+\alpha_{i 2} q\left(\alpha_{i 1}, \alpha_{i 2}\right.$ in $\left.K\right)$, we shall have $Q=0$ for $\alpha_{i}$ in $J[q]$, that is, $\alpha_{i 1}$ and $\alpha_{i 2}$ in $J$. But then

$$
Q=\sum \lambda_{i}\left[\left(\alpha_{i 1}{ }^{2}+\alpha_{i 2}{ }^{2} \delta\right)+\left(2 \alpha_{i 1} \alpha_{2 i}\right) q\right]=0
$$

so that

$$
\sum \lambda_{i} S_{i}=0,
$$

where

$$
S_{i}=\left(\alpha_{i 1}\right)^{2}+\left(\alpha_{i 2} \delta_{1}\right)^{2}+\left(\alpha_{i 2} \delta_{2}\right)^{2} .
$$

We shall call a polynomial of the form (22) an $S$-polynomial. All such polynomials have the properties that all their degrees are even, all their ( , , )-leading coefficients positive. Moreover such a polynomial is zero if and only if $\alpha_{i}=\alpha_{i 1}=\alpha_{i 2}=0$. Hence we have 
Lemma 3. A sufficient condition that a quadratic form $\sum \lambda_{i} \alpha_{i}{ }^{2}$ with $\lambda_{i}$ in $J$ shall not vanish for any $\alpha_{i}$ not all zero and in $K(q)$ is that $\sum \lambda_{i} S_{i}$ shall not vanish for any $S$-polynomials $S_{i}$ not all zero.

6. The multiplication constants of $A$. We now choose $\rho, \sigma, \gamma_{1}, \cdots, \gamma_{6}$ in $J$. We shall take

$$
\sigma \text { of even } z \text {-degree, even }(z, y) \text {-degree, odd }(z, y, x) \text {-degree. }
$$

We shall define $\gamma_{1}$ and $\gamma_{5}$ in terms of certain quantities $\epsilon_{1}, \epsilon_{5}$, where

$$
\begin{aligned}
& \text { (the } \left.z \text {-degree of } \epsilon_{5} \text { is odd }\right)>\left(z \text {-degree of } \epsilon_{1} \gamma_{3}\right) ; \\
& \text { the } \left.z \text {-degree of } \gamma_{3} \text { is odd }\right)>\left(z \text {-degree of } \gamma_{4} \sigma\right) ; \\
& \text { the } \left.z \text {-degree of } \gamma_{2}\right)>\left(z \text {-degree of } \gamma_{6} \sigma\right) ; \\
& \text { the }(z, y) \text {-degree of } \gamma_{3} \text { even, of } \epsilon_{5} \text { odd. }
\end{aligned}
$$

The above conditions are restrictions merely on the z-leading coefficients of our quantities. By making the corresponding $z$-degrees sufficiently large we evidently only restrict a single term in each quantity, satisfy the above conditions, and yet permit any desired inequalities between $x$-degrees, $y$-degrees of the same quantities. Moreover ( , , )-leading coeffcients other than the $(z, \quad, \quad)$-leading coefficients may be taken to have any desired sign, and the evenness or oddness of ( , )-degrees, etc., other than those already given above are still at our choice. We therefore may continue with

$$
\begin{aligned}
& \sigma \text { of even } y \text {-degree, odd. }(y, x) \text {-degree; } \\
& \left(y \text {-degree of } \epsilon_{1} \text { odd }\right)>\left(y \text {-degree of } \epsilon_{5}\right) ; \\
& \left(y \text {-degree of } \gamma_{2}\right)>\left(y \text {-degree of } \gamma_{6} \sigma\right) ; \\
& \left(y \text {-degree of } \gamma_{3}\right)>\left(y \text {-degree of } \gamma_{4} \sigma\right) ; \\
& \sigma \text { of odd } x \text {-degree. }
\end{aligned}
$$

Let the $x$-leading coefficient of $\gamma_{6}$ be $\pi_{1}$, that of $\gamma_{2} \gamma_{4}$ be $\pi_{2}$ such that

$$
\pi_{1}^{2}+\pi_{2}^{2} \neq \lambda^{2} \text { for any } \lambda \text { of } F(y, z) \text {. }
$$

This restriction may be satisfied by Lemma 2 and there merely restricts the $x$-leading coefficients of $\gamma_{6}$ and $\gamma_{2} \gamma_{4}$. Also take

$$
\left(x \text {-degree of } \gamma_{6}\right)=\left(x \text {-degree of } \gamma_{2} \gamma_{4}\right)>\left(x \text {-degree of } \gamma_{2} \gamma_{3}\right) \text {, }
$$

that is, the $x$-degree of $\gamma_{4}$ greater than the $x$-degree of $\gamma_{3}$, and, if we desire, the $x$-leading coefficient of $\gamma_{2}$ unity, that of $\gamma_{4}, y$, that of $\gamma_{6}, z$, and (33) is satisfied. 
Finally let

$$
\begin{aligned}
e & =\gamma_{2}^{2}\left(\gamma_{3}^{2}-\gamma_{4}^{2} \sigma\right)-\gamma_{6}^{2} \sigma, \\
\rho & =e\left[\epsilon_{1}^{2}\left(\gamma_{3}^{2}-\gamma_{4}^{2} \sigma\right)-\epsilon_{5}^{2}\right], \\
\gamma_{1} & =\epsilon_{1} e, \quad \gamma_{5}=\epsilon_{5} e .
\end{aligned}
$$

Then

$$
\begin{aligned}
\gamma_{1}{ }^{2}-\gamma_{2}{ }^{2} \rho & =\epsilon_{1}{ }^{2} e^{2}-\gamma_{2}{ }^{2} \rho \\
& =e \epsilon_{1}{ }^{2}\left[\gamma_{2}{ }^{2}\left(\gamma_{3}{ }^{2}-\gamma_{4}{ }^{2} \sigma\right)-\gamma_{6}{ }^{2} \sigma\right]-e \gamma_{2}{ }^{2} \epsilon_{1}{ }^{2}\left(\gamma_{3}{ }^{2}-\gamma_{4}{ }^{2} \sigma\right)+\gamma_{2}{ }^{2} \epsilon_{5}{ }^{2} e,
\end{aligned}
$$

and

$$
\gamma_{1}^{2}-\gamma_{2}^{2} \rho=e\left[\left(\gamma_{2} \epsilon_{5}\right)^{2}-\left(\gamma_{6} \epsilon_{1}\right)^{2} \sigma\right]
$$

Also

$$
\begin{aligned}
\gamma_{5}{ }^{2}-\gamma_{6}{ }^{2} \sigma \rho & =\epsilon_{5}{ }^{2} e^{2}-\gamma_{6}{ }^{2} \sigma \rho \\
& =e \gamma_{2}{ }^{2} \epsilon_{5}{ }^{2}\left(\gamma_{3}^{2}-\gamma_{4}{ }^{2} \sigma\right)-e \gamma_{6}{ }^{2} \epsilon_{5}{ }^{2} \sigma+e \gamma_{6}{ }^{2} \sigma \epsilon_{5}{ }^{2}-e \gamma_{6}{ }^{2} \sigma \epsilon_{1}{ }^{2}\left(\gamma_{3}{ }^{2}-\gamma_{4}{ }^{2} \sigma\right) \\
& =\left(\gamma_{3}{ }^{2}-\gamma_{4}{ }^{2} \sigma\right) e\left[\left(\gamma_{2} \epsilon_{5}\right)^{2}-\left(\gamma_{6} \epsilon_{1}\right)^{2} \sigma\right] .
\end{aligned}
$$

By (38) we have

TheoRem 4. If $\rho, \sigma, \gamma_{1}, \cdots, \gamma_{6}$ are chosen as in (35), (36), (37), the corresponding algebra $A$ satisfies

$$
\gamma_{5}^{2}-\gamma_{6}^{2} \sigma \rho=\left(\gamma_{1}^{2}-\gamma_{2}^{2} \rho\right)\left(\gamma_{3}^{2}-\gamma_{4}^{2} \sigma\right)
$$

and is associative.

7. Elementary properties. In (25) we chose the $z$-degree of $\gamma_{3}$ to be greater than the $z$-degree of $\gamma_{4} \sigma$. In (26) we took the $z$-degree of $\gamma_{2}$ greater than that of $\gamma_{6} \sigma$. It now follows that the only term of $e$ containing its highest power of $z$ is $\left(\gamma_{2} \gamma_{3}\right)^{2}$. Similarly, by (24), (25) the term of $\left[\epsilon_{1}{ }^{2}\left(\gamma_{3}^{2}-\gamma_{4}^{2} \sigma\right)-\epsilon_{5}{ }^{2}\right]$ containing its highest power of $z$ is $-\epsilon_{5}{ }^{2}$. Hence the term of $\rho$ containing its highest power of $z$ is $-\left(\gamma_{2} \gamma_{3} \epsilon_{5}\right)^{2}$.

Lемма 4. The z-degree of $\rho$ is positive, even, and the z-leading coefficient of $\rho$ is the negative of a perfect square.

Consider the $y$-degree of $\rho$. By (31) the $y$-degree of $\gamma_{3}^{2}-\gamma_{4}^{2} \sigma$ is positive and its $y$-leading coefficient is a perfect square (in $\gamma_{3}^{2}$ ). By (35) the leading $y$-term of $e$ is then in $\left(\gamma_{2} \gamma_{3}\right)^{2}$, while the leading $y$-term of $\epsilon_{1}^{2}\left(\gamma_{3}^{2}-\gamma_{4}^{2} \sigma\right)-\epsilon_{5}^{2}$ is then in $\left(\epsilon_{1} \gamma_{3}\right)^{2}$. Hence the term of $\rho$ containing its highest power of $y$ is $\left(\epsilon_{1} \gamma_{2} \gamma_{3}^{2}\right)^{2}$.

LEMMA 5. The $y$-degree of $\rho$ is positive and even, and its y-leading coefficient is a perfect square. 
Consider the $x$-degree of $e$. We have taken the $x$-degree of $\gamma_{6}$ equal to the $x$-degree of $\gamma_{2} \gamma_{4}$ and the $x$-degree of $\gamma_{4}$ greater than the $x$-degree of $\gamma_{3}$. But $e=-\left[\left(\gamma_{2} \gamma_{4}\right)^{2}+\gamma_{6}{ }^{2}\right] \sigma+\left(\gamma_{2} \gamma_{3}\right)^{2}$. Hence the $x$-leading coefficient of $e$ is the product of the $x$-leading coefficient of $-\sigma$ by $\pi_{1}^{2}+\pi_{2}^{2}$. But the $x$-degree of $\sigma$ has been taken odd.

LEMмA 6. Let $\sigma_{0}$ be the $x$-leading coefficient of $\sigma$. Then the $x$-leading coefficient of $e$ is $-\sigma_{0}\left(\pi_{1}^{2}+\pi_{2}^{2}\right)$ and the $x$-degree of $e$ is a positive odd integer.

The quantity $\gamma_{1}^{2}-\gamma_{2}^{2} \rho$ is determined by (38). We shall require

LEMMA 7. The z-degrees of $\gamma_{1}^{2}-\gamma_{2}^{2} \rho$ are all even.

For proof we notice that we have already shown that the $z$-degree of $e$ is even, in fact the leading term of $e$ when arranged according to powers of $z$ is a perfect square. Also we have taken the $z$-degree of $\left(\gamma_{2} \epsilon_{5}\right)^{2}$ greater than that of $\left(\gamma_{6} \epsilon_{1}\right)^{2} \sigma$. Hence the $z$-degree of $\gamma_{1}^{2}-\gamma_{2}^{2} \rho$ is even. In fact its $z$-leading coefficient occurs only in $\left(\gamma_{2}^{2} \epsilon_{5} \gamma_{3}\right)^{2}$ and is a perfect square, so that all its $z$-degrees are even.

One of the properties required in our definition of $A$ is that neither $\rho, \sigma$, nor $\sigma \rho$ shall be the square of any quantities of $K$. We shall prove

LEMMA 8. Neither $\rho, \sigma$, nor $\sigma \rho$ is the square of any quantity of $K(q)$.

For let $\rho=\alpha^{2}$ where $\alpha$ is in $K(q)$. Then $\mu \alpha=\lambda$ where $\lambda$ is in $J[q]$ and $\mu$ is in $J$. Then $\rho \mu^{2}=\lambda^{2}$ in $J$. A quantity $\lambda$ of $K(q)$ has its square in $K$ if and only if it is either in $K$ or a multiple of $q$ by a quantity of $k$. If $\lambda$ in $J[q]$ is in $K$ then $\lambda$ is in $J$ so that $\rho \mu^{2}=\lambda^{2}$ is impossible because the $(z, y, x)$-leading coefficient of $\rho$ and hence $\rho \mu^{2}$ is negative while that of $\lambda^{2}$ is positive. Hence $\lambda=\nu q$ with $\nu$ in $J$. Then $\lambda^{2}=\nu^{2} \delta$ is an $S$-polynomial and cannot be identical with $\rho \mu^{2}$ of negative $(z, y, x)$-leading coefficient.

Similarly $\sigma \neq \alpha^{2}$ where we now use the property that $\sigma$ has odd $x$-degree. Finally by (28) and Lemma $5 \sigma \rho$ has odd $(y, x)$-degree and $\sigma \rho \neq \alpha^{2}$ for any $\alpha$ of $K(q)$.

COROLLARY 1. The quantities $\rho, \sigma, \sigma \rho$ are not the squares of any quantities of $K$.

It follows from Corollary 1 that $K(u, v)$ is a quartic field over $K$ and that $g_{1}=0$ if and only if $\gamma_{1}=\gamma_{2}=0$. By Lemma $7, g_{1} \neq 0$. Also (31) implies that $g_{2} \neq 0$, while the associativity condition (38) implies that $g_{3} \neq 0$.

8. The exponent of $A$. We shall use (7) to prove that $A$ has exponent four, that is, $A$ is not a direct product of two algebras of degree two. Assume that $A$ has not exponent four so that (7) is satisfied for $\alpha_{1}, \alpha_{2}, \alpha_{3}$ in $K$ and not all zero. As we have already remarked we may take $\alpha_{1}, \alpha_{2}, \alpha_{3}$ in $J$. If $\alpha_{2}=\alpha_{3}=0$, 


$$
\alpha_{1}^{2}-\alpha_{2}^{2} \sigma=\left(\gamma_{1}^{2}-\gamma_{2}^{2} \rho\right) \alpha_{3}^{2}
$$

implies that $\alpha_{1}^{2}=\alpha_{1}=0$, a contradiction. Hence if $\alpha_{3}=0$ then $\alpha_{2} \neq 0$ and $\sigma=\left(\alpha_{1} \alpha_{2}^{-1}\right)^{2}$, a contradiction of Corollary 1. Thus $\alpha_{3} \neq 0$.

By Lemma $7 \gamma_{1}^{2}-\gamma_{2}^{2} \rho \neq 0$ so that $h=\left(\gamma_{2} \epsilon_{5}\right)^{2}-\left(\gamma_{6} \epsilon_{1}\right)^{2} \sigma \neq 0$. The equation $\gamma_{1}^{2}-\gamma_{2}^{2} \rho=h e$ gives

$$
\left(\alpha_{1}^{2}-\alpha_{2}^{2} \sigma\right) l==\left(\alpha_{3} h\right)^{2} e .
$$

Let $\beta_{3}=\alpha_{3} h \neq 0, \beta_{1}=\alpha_{1} \gamma_{2} \epsilon_{5}+\alpha_{2} \gamma_{6} \epsilon_{1} \sigma, \beta_{2}=\alpha_{1} \gamma_{6} \epsilon_{1}+\alpha_{2} \gamma_{2} \epsilon_{5}$. Then, as may be easily computed, ${ }^{*}$

$$
\beta_{1}^{2}-\beta_{2}^{2} \sigma=e \beta_{3}^{2} \quad\left(\beta_{3} \neq 0, \beta_{1}, \beta_{2}, \beta_{3} \text { in } J\right) .
$$

But then $\beta_{1}^{2}=\sigma \beta_{2}^{2}+e \beta_{3}^{2}$. The $x$-leading coefficient of $e \beta_{3}{ }^{2}$ has the form $-\sigma_{0}\left(\pi_{1}^{2}+\pi_{2}^{2}\right) \beta_{30}{ }^{2}$ by Lemma 6 . The $x$-leading coefficient of $\sigma \beta_{2}^{2}$ has the form $\sigma_{0} \beta_{20}{ }^{2}$. But $\left(\pi_{1}^{2}+\pi_{2}^{2}\right) \beta_{30}^{2} \neq 0$ is not the square of any quantity of $K(y, z)$. Hence the $x$-leading coefficient of $\sigma{\beta_{2}}^{2}+e \beta_{3}{ }^{2}$ is not zero. But the $x$-degree of this expression is odd since $\sigma$ has odd $x$-degree, $e$ has odd $x$-degree, $\beta_{3} \neq 0$. It follows that (40) is impossible for $\beta_{3} \neq 0$, a contradiction.

9. The first norm condition. We wish to prove that algebra $B$ is a division algebra, that is, prove that $g_{1} \neq a \cdot a(-v)$ for any $a$ of $K(u, v)$, the so called first norm condition. As we have shown this condition will be satisfied if we can show that the equation

$$
S_{1}+S_{2} \rho-\sigma\left(S_{3}+S_{4} \rho\right)=\gamma_{1} S_{5}
$$

is impossible for $S$-polynomials $S_{1}, \cdots, S_{5}$ not all zero, a consequence of $\$ 5$ applied to (9).

By Lemma 2 the $y$-degree of $\rho$ is even and the $(y, z, x)$-leading coefficient of $\rho$ is positive. Also the $y$-degree of $\sigma$ is even. Hence the $y$-degree of each of $S_{1}, S_{2} \rho, S_{3}, S_{4} \rho$ is even. But the $(y, z, x)$-leading coefficients of these terms are all positive. Moreover $S_{1}+S_{2} \rho, S_{3}+S_{4} \rho$ have even $(y, z)$-degree, while $\sigma$ has odd $(y, z)$-degree. Hence the $(y, z)$-degree of $S_{1}+S_{2} \rho-\sigma\left(S_{3}+S_{4} \rho\right)$ is either even or odd according as the $(y, z)$-degree of $S_{1}+S_{2} \rho$ is greater or less than the $(y, z)$-degree of $\left(S_{3}+S_{4} \rho\right) \sigma$. In any case the corresponding $(y, z, x)$-leading coefficient is zero if and only if $S_{1}=S_{2}=S_{3}=S_{4}=0$. We have shown that $T=S_{1}+S_{2} \rho-\sigma\left(S_{3}+S_{4} \rho\right)$ has even $y$-degree and $(y, z, x)$-leading coefficient zero if and only if $S_{i}=0(i=1, \cdots, 4)$.

By (35), (30), (31) the $y$-degree of $e$ is even. By (37), (29) the $y$-degree of $\gamma_{1}$ is odd. Hence the $y$-degree of $\gamma_{1} S_{5}$ is odd unless $S_{5}=0$. But $\gamma_{1} S_{5}=T$ has even $y$-degree. Hence $S_{5}=0, T=0, T$ has $(y, z, x)$-leading coefficient zero so that $S_{i}=0(i=1, \cdots, 5)$.

* That is, let $a=\alpha_{1}+\alpha_{2} v, b=\gamma_{2} \epsilon_{5}+\gamma_{6} \epsilon_{1} v$. Then $a b=\left(\alpha_{1} \gamma_{2} \epsilon_{5}+\alpha_{2} \gamma_{6} \epsilon_{1} \sigma\right)+\left(\alpha_{1} \gamma_{6} \epsilon_{1}+\alpha_{2} \gamma_{2} \epsilon_{b}\right) v=\beta_{1}+\beta_{2} v$, and $a \cdot a(-v) \cdot b \cdot b(-v)=\left(\alpha_{1}^{2}-\alpha_{2}^{2} \sigma\right) \cdot h=a b \cdot \overline{a b}(-v)=\beta_{1}^{2}-\beta_{2}^{2} \sigma$. 
10. The second norm condition. This is the condition $g_{2}=X^{\prime} X$ which, by $\$ 5$ and (19), is satisfied if we can prove that

$$
\gamma_{5}\left[S_{1}+S_{2} \sigma-\rho\left(S_{3}+S_{4} \sigma\right)\right]+\gamma_{3}\left(\gamma_{1}^{2}-\gamma_{2}^{2} \rho\right)\left[S_{5}+S_{6} \sigma \rho-\rho S_{7}-\sigma S_{8}\right]=\gamma_{3} \gamma_{5} S_{9}
$$

is impossible for $S$-polynomials $S_{i}(i=1, \cdots, 9)$ not all zero. Notice that we have replaced $\rho \alpha_{8}^{2} \rho=\left(\rho \alpha_{8}\right)^{2}$ of (19) by the $S$-polynomial $S_{8}$ instead of the formally corresponding $\rho^{2} S_{8}$.

By (24) the $z$-degree of $\gamma_{3}$ is odd. By the proof of Lemma 4 the $z$-degree of $e$ is even and the $z$-leading coefficient of $e$ is a perfect square. Applying (27) we have

\section{LEMMA 9. The $z$ - and $(z, y)$-degrees of $\gamma_{b}$ are odd.}

We have taken $\rho$ to have all even degrees and negative $(z, y, x)$-leading coefficient by Lemma 4 . Also $\sigma$ has even $z$-degree, $(z, y)$-degree, but odd $(z, y, x)$-degree. Hence the $(z, y, x)$-leading coefficient of any $S_{i}-\rho S_{j}$ is positive or zero according as not both or both of $S_{i}, S_{j}$ are zero. Hence the $(z, y, x)$-leading coefficient of a combination $T=S_{i}-\rho S_{j} \pm \sigma\left(S_{r}-\rho S_{t}\right)$ is zero if and only if the four $S_{i}$ are zero. Moreover $T$ has even $(z, y)$-degree and $(z, y)$-leading coefficient which is identically zero only when all the four $S_{i}$ are zero. But the $(z, y)$-degree of $\gamma_{3}$ is even, the $(z, y)$-degree of $\gamma_{1}^{2}-\gamma_{2}^{2} \rho$ is even, while that of $\gamma_{5}$ is odd. Hence the $(z, y)$-leading coefficient of

$$
R=\gamma_{5}\left[\left(S_{1}-\rho S_{3}\right)+\sigma\left(S_{2}-\rho S_{4}\right)\right]+\gamma_{3}\left(\gamma_{1}^{2}-\gamma_{2}^{2} \rho\right)\left[S_{5}-\rho S_{7}-\sigma\left(S_{6}-\rho S_{8}\right)\right]
$$

is either the $(z, y)$-leading coefficient of its first bracket or of its second bracket, while $R$ has z-leading coefficient identically zero if and only if $S_{i}=0$ $(i=1, \cdots, 8)$. But the $z$-degree of $R$ is odd unless the $S_{i}$ are zero since the $z$-degree of $\gamma_{3}$ is odd by (25), that of $\gamma_{5}$ odd by Lemma 9. By (42) $R=\gamma_{3} \gamma_{5} S_{9}$ has even z-degree. Hence $R=0, S_{9}=0$, and $R$ has z-leading coefficient zero. This proves that $S_{i}=0(i=1, \cdots, 9)$ as desired. We have proved

LEMMA 10. Let $F$ be a real number field, $x, y, z$ indeterminates, and let $A$ be an algebra of order sixteen over $K=F(x, y, z)$ defined by (1)-(5), (23)-(37). Then $A$ is a normal division algebra of degree and exponent four over $K, A \times L$ is a normal division algebra of degree four over $L$ for every quadratic field $L=K(q), q^{2}=\delta=\delta_{1}^{2}+\delta_{2}^{2}\left(\delta_{1}, \delta_{2}\right.$ in $\left.K\right)$.

As an immediate corollary of Lemma 10 we then have

Theorem. The algebras of Lemma 10 are non-cyclic algebras of degree four not expressible as direct products of cyclic algebras of degree two.

University of Chicago, Chicago, Ill. 